\section{Purpose and Significance of the 2010 Criteria and Modification of the 2010 Criteria for Fibromyalgia}

\section{To the Editor:}

I read a reply from Dr. F. Wolfe ${ }^{1}$. I did not misunderstand. I understand that modification of the American College of Rheumatology (ACR) preliminary diagnostic criteria for fibromyalgia (FM) are not the ACR criteria. I did not state that the modification of the ACR preliminary criteria were the ACR criteria ${ }^{1}$. Dr. Wolfe, et al stated as follows: ACR preliminary diagnostic criteria for FM in 2010 eliminated the tender point examination, thus making it possible to study FM in survey and clinical research ${ }^{2}$. A modification to the ACR criteria will allow their use in epidemiologic and clinical studies without the requirement for an examiner ${ }^{2}$. Therefore, I had believed that the modification of the 2010 criteria had replaced the 2010 criteria ${ }^{1}$. Is it due to my misunderstanding or due to the description by Dr. Wolfe, et al? I accept a conclusion of Dr. Wolfe: The modification of the 2010 criteria is designed for survey research only (survey criteria) ${ }^{1}$. Are the 2010 criteria used for individual diagnosis only (clinical criteria)? I sometimes perform a cross-sectional study or retrospective study with individually diagnosed patients with FM. In this case, which criteria should be used?

Dr. Wolfe stated that the proposed survey criteria (the modification of the 2010 criteria) did not include FM symptom score ${ }^{1}$. They showed a sensitivity and specificity of the FM symptom score $\geq 13^{2}$. Dr. Wolfe, et al stated that their scales were designed primarily for aiding in diagnosis ${ }^{2}$. Their scales mean FM symptom scale (FM symptom score) and symptom severity scale ${ }^{2}$. What is the purpose of FM symptom scale (or FM symptom score)?

Physicians have to investigate symptom severity scale score in the 2010 criteria $^{2}$. The time for diagnosis with the 2010 criteria including time for explanation of some terms such as Raynaud's phenomenon may be longer than that with the 1990 criteria. It is a disadvantage of the 2010 criteria. Even if physicians investigate symptom severity score in the modification of the 2010 criteria, the time for diagnosis with the 2010 criteria is longer than that with the modification of the 2010 criteria. I am afraid that not a few physicians may use the modification of the 2010 criteria for indi- vidual diagnosis because of its short duration of diagnosis. Frequently used diagnostic criteria are substantive diagnostic criteria.

I am afraid that clinical criteria for FM have little or no significance from the viewpoint of clinical practice. Chronic regional pain and chronic widespread pain are usually an incomplete form of $\mathrm{FM}^{3}$. Chronic regional pain, chronic widespread pain, and FM are thought to be a continuous disorder. Treatment of chronic widespread pain is usually identical with treatment of FM throughout the world ${ }^{3}$. Natelson stated that treatment for patients with chronic regional pain was the same as it was for patients with $\mathrm{FM}^{4}$. If treatments of 2 disorders are the same, differentiation between the 2 disorders provides little or no significance from the viewpoint of clinical practice. Diagnostic criteria are useful in a conference presentation and/or in writing articles alone.

KATSUHIRO TODA, MD, PhD, Department of Rehabilitation, Hatsukaichi Memorial Hospital, Hatsukaichi, 5-12 Youkoudai, Hatsukaichi, Hiroshima 738-0060, Japan. E-mail: goutattack@yahoo.co.jp

\section{REFERENCES}

1. Toda K. The modification of the American College of Rheumatology preliminary diagnostic criteria for fibromyalgia should be supplemented and revised [letter]. J Rheumatol 2011;38:2075; reply 2075-6.

2. Wolfe F, Clauw DJ, Fitzcharles MA, Goldenberg DL, Hauser W, Katz RS, et al. Fibromyalgia criteria and severity scales for clinical and epidemiological studies: A modification of the ACR preliminary diagnostic criteria for fibromyalgia. J Rheumatol 2011;38:1113-22

3. Toda K. Treatment of chronic widespread pain is similar to treatment of fibromyalgia throughout the world. J Musculoskelet Pain 2010;18:317-8.

4. Natelson BH. Fibromyalgia syndrome: Diagnosis and comorbidities. CNS Spectr 2009;14:8-11; discussion 17-8.

J Rheumatol 2012;39:6; doi:10.3899/jrheum.120094 